\title{
Modeling of graceful motions: determining characteristics of graceful motions from handover motion
}

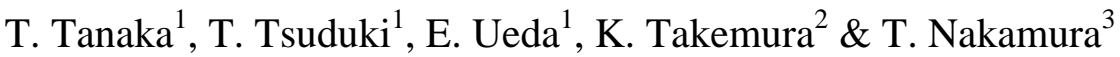 \\ ${ }^{1}$ Nara National College of Technology, Japan \\ ${ }^{2}$ Nara Institute of Science and Technology, Japan \\ ${ }^{3}$ Wakayama University, Japan
}

\begin{abstract}
Since robots in the welfare and service industries make contact with humans, they have to produce favorable impressions in the human mind. This study proposes graceful motions of humans and aims to quantify it to make robot motions more favorable. We have focused on the handover motion of a glass and selected waiters as subjects. Their motions were recorded as trajectories by motion capture system and evaluated by observers. The result revealed that the motions of them produce favorable and graceful impressions. The trajectory was projected on a two-dimensional plane surface that was obtained by conducting principal component analysis. The projected trajectory was calculated by fitting 2 spline curves. As a result, the common characteristics of graceful motions were extracted as parameters of spline curves and graceful motions are characterized by S-shaped trajectories. After that, 4 motions were created using 3DCG by changing extracted parameters step by step to verify which parameters provide graceful impressions through simulations. Finally, the parameters that produce graceful and favorable impressions were determined.
\end{abstract}

Keywords: graceful motions, motion analysis, motion capture.

\section{Introduction}

Nowadays, robot systems are finding widespread applications not only in industry but also in the welfare and service industries. However, the mechanical motions of typical industrial robots may produce unfavorable impressions in the human mind because they are geared toward performing tasks efficiently. Now, 
robots in the welfare and service industries make contact with humans, and therefore, the impressions made by robot motions are more important than efficiency. In this light, it is important to enable these robots to perform humanlike motions and produce favorable impressions in the human mind.

People's experiences suggest that robot motions can produce favorable impressions if they are human-like. Abu bakar et al. [1] focused on the cooperative motion of two humans and reported characteristics of human-like motion. Moreover, Yokoi et al. [2] investigated a robot motion that can produce kind impressions in human mind. Many researchers have investigated about human-like motions. However, human-like motions have not been defined clearly. At the same time, recently, the concept of "grace" has been discussed in various research areas. Meinel [3] stated that "the grace of motions provides comfort and good impressions. Moreover it inspires elegant impressions" and that "the grace of motions is divided into two categories, natural grace and conscious grace." This suggests that graceful motions can produce favorable impressions in the human mind. Furthermore, we can consciously make motions graceful. Schiller [4] stated that "grace is beauty of action and can be applied to unbeautiful appearances." This suggests that any kind of appearances can be made graceful. And, the "graceful" is inherent adjective of human motion. Therefore, we considered that robot motions look like human by being made graceful.

This study aimed to quantify the definitions presented in literature and to model graceful motions so as to make robots motions more favorable. Fig. 1 shows the flow of this study.

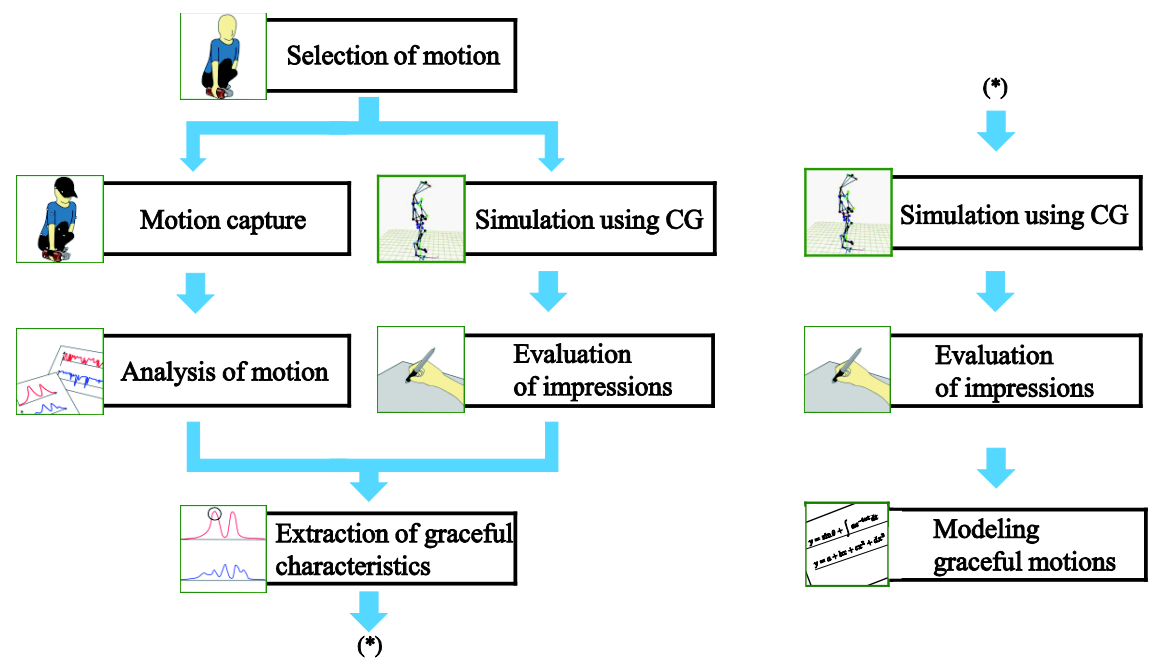

Figure 1: $\quad$ Flow of this study. 


\section{Graceful motions}

Meinel's and Schiller's thoughts have already been mentioned above. Furthermore, Hogarth [5] discussed "The line of beauty." This suggests that the S-shaped curved lines (serpentine lines) signify beauty and grace and S-shaped hand motions appear graceful. Buytendijk [6], who had studied kinesiology in terms of phenomenological anthropology, stated that graceful motions have the following characteristics:

1. Rapid and rectilinear motions are angulated motions and therefore do not appear graceful. On the other hand, slow and rounded motions appear graceful.

2. Discontinuous motions do not appear graceful. On the other hand, continuous and fluid motions appear graceful.

3. Monotonously repeating motions do not appear graceful. On the other hand, motions that rhythmically alternate between contraction and relaxation appear graceful.

In this study, we employ these characteristics to define graceful motions, and we investigate whether motions perceived as being graceful possess these characteristics.

\section{Analysis of handover motion of a glass}

In this study, we have focused on the handover motion of a glass. We selected waiters who work at a hotel as subjects because they are very particular about their mannerisms and are trained to produce favorable impressions in the minds of customers.

\subsection{Evaluation of impressions}

In our experiment, eight people - four of whom were trained at a special school for front desk clerks, bell-boys, waiters, and waitresses (Subject1-3: men, Subject4: woman) and the other four of whom were ordinary people (Subject58: men) - participated as subjects. For the evaluation, eight movies were created using 3DCG to eliminate prejudice. The ten observers evaluated the impressions of each motion. Fig. 2 shows an example of the handover motion. The movies were evaluated against five pairs of adjectives, shown in Fig. 3(a), using the semantic differential method. Fig. 3(b) shows the average scores of each subject; it was found that the trained subjects received higher scores than ordinary subjects for motions related to grace. Therefore, we considered the motions of the trained and the ordinary subjects as graceful and general motions, respectively. 


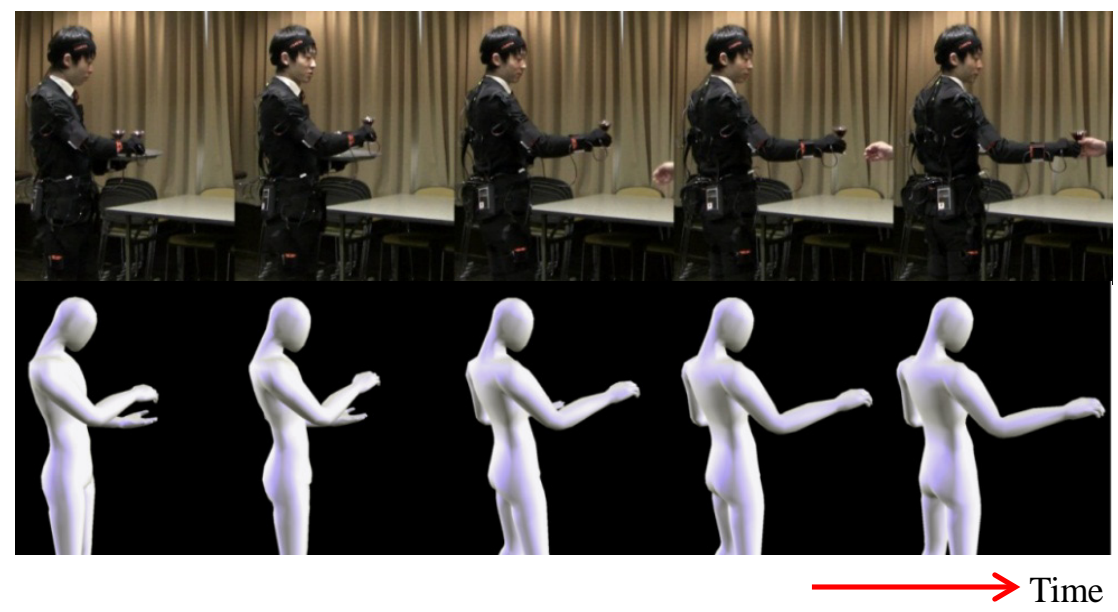

Figure 2: $\quad$ Example of the handover motion.

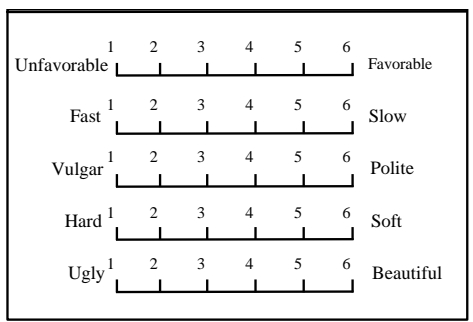

(a) 5 pairs of adjectives.

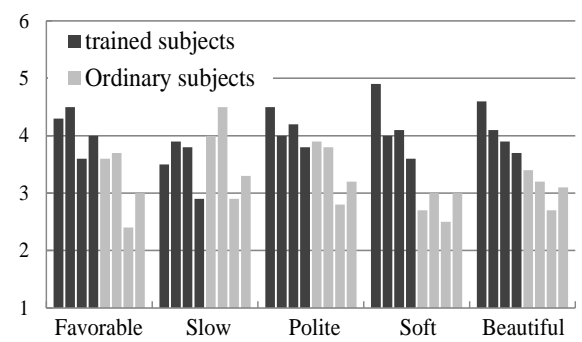

(b) Average scores of each subject.

Figure 3: $\quad$ Evaluation of impressions.

\subsection{Ellipsoid fitting}

Accordingly, the direction of motions and the height direction are respectively considered as the $\mathrm{x}$ - and the y-axis. Furthermore, the coordinate system is considered a right-handed coordinate system.

In our previous study, Tsuduki et al. [7] projected the trajectory of each ordinary subject onto the $\mathrm{x}-\mathrm{y}$ plane and calculated by fitting an ellipse. In this study, an ellipsoid was fitted to each three-dimensional trajectory. Considering a semi-major axis and a semi-minor axis of the ellipsoid as $a$ and $b$ respectively, table 1 shows the parameters of the ellipsoid whose error is the smallest. The result revealed that the errors of Subject1, Subject2, and Subject3 were high. Therefore, the trajectories of their motions are not elliptical and differ from those of ordinary subjects. However, the result of Subject 4 among the trained subjects was the same as that of the ordinary subjects. This result suggests that a difference may exist between men and women. 
Table 1: $\quad$ Result of ellipsoid fitting.

\begin{tabular}{|c|c|c|c|c|c|}
\hline & & $a[\mathrm{~cm}]$ & $b[\mathrm{~cm}]$ & $a / b$ & Error[\%] \\
\hline \multirow{4}{*}{$\begin{array}{c}\text { Trained } \\
\text { subjects }\end{array}$} & Subject1 & 14.6 & 3.0 & 0.21 & 47.6 \\
\cline { 2 - 6 } & Subject2 & 18.1 & 8.5 & 0.47 & 35.7 \\
\cline { 2 - 6 } & Subject3 & 22.1 & 2.5 & 0.11 & 36.0 \\
\cline { 2 - 6 } & Subject4 & 15.1 & 6.6 & 0.44 & 3.2 \\
\hline \multirow{4}{*}{$\begin{array}{c}\text { Ordinary } \\
\text { subjects }\end{array}$} & Subject5 & 15.9 & 6.1 & 0.39 & 8.7 \\
\cline { 2 - 6 } & Subject6 & 13.4 & 7.5 & 0.56 & 6.6 \\
\cline { 2 - 6 } & Subject7 & 7.3 & 4.4 & 0.60 & 6.8 \\
\cline { 2 - 6 } & Subject8 & 7.0 & 4.3 & 0.61 & 15.6 \\
\hline
\end{tabular}

\subsection{Fitting of spline curves}

The trajectories of the motions of Subject1, Subject2, and Subject3 are not elliptical. Therefore, we focused on a plane surface on which each subject performs motions. The plane surface was extracted by conducting principal component analysis. A plane surface that consists of the first principal component and the second principal component was defined as the motion characteristics plane surface. Then, the principal component score on this surface was obtained as a two-dimensional trajectory.

This trajectory was fitted by two spline curves. The cubic spline curves are expressed by the following equation:

$$
y=a+b x+c x^{2}+d x^{3}
$$

When the trajectory was fitted, the position of one-tenth and nine-tenth were respectively considered as $\mathrm{P} 1$ and $\mathrm{P} 3$. Between P1 and P3, P2 was changed and spline interpolation was conducted using a starting point, P1, P2, P3, and an end point. Fig. 4 (a) shows an example of the motion characteristics plane surface. Fig. 4 (b) shows an example of the fitting of spline curves. As shown in fig. 4 (a), the motion characteristics plane surface leans against the $\mathrm{x}-\mathrm{z}$ plane. Considering the angle between this surface and the $\mathrm{x}-\mathrm{z}$ plane as $\theta$, table 2 shows the results of spline fitting whose error is the smallest. P2 is the value in case in which the value between $\mathrm{P} 1$ and $\mathrm{P} 3$ is considered as 1 . As shown in table 2, the $\theta$ values of Subject1, Subject2, and Subject3 are smaller than those of ordinary subjects; therefore, graceful motions were mainly performed in crosswise directions. On the other hand, the $\theta$ values of Subject5, Subject6, Subject7, and Subject8 are large; therefore, general motions were mainly performed in vertical directions. Moreover, the $b, c$, and $d$ values of Subject1, Subject2, and Subject3 are almost the same, and P2 is positioned in the first half of the trajectory. This means that their trajectories have the same shape. 
Table 2: $\quad$ Parameters of spline curves.

\begin{tabular}{|c|c|c|c|c|c|c|c|}
\hline & & $\theta\left[^{\circ}\right]$ & $\mathrm{S}$ & $b$ & $c$ & $d$ & $\mathrm{P} 2$ \\
\hline \multirow{8}{*}{$\begin{array}{l}\text { Trained } \\
\text { subjects }\end{array}$} & \multirow{2}{*}{ Subject1 } & \multirow{2}{*}{30.4} & S1 & -1.1 & 0.2 & -0.011 & \multirow{2}{*}{0.23} \\
\hline & & & $\mathrm{S} 2$ & 0.11 & 0.013 & -0.00041 & \\
\hline & \multirow{2}{*}{ Subject2 } & \multirow{2}{*}{43.7} & S1 & -0.8 & 0.14 & -0.0089 & \multirow{2}{*}{0.11} \\
\hline & & & $\mathrm{S} 2$ & -0.11 & 0.014 & -0.00018 & \\
\hline & \multirow{2}{*}{ Subject3 } & \multirow{2}{*}{4.8} & $\mathrm{~S} 1$ & 3.23 & -0.54 & 0.028 & \multirow{2}{*}{0.23} \\
\hline & & & $\mathrm{S} 2$ & -0.22 & -0.036 & 0.0012 & \\
\hline & \multirow{2}{*}{ Subject4 } & \multirow{2}{*}{70.5} & $\mathrm{~S} 1$ & -0.56 & 0.028 & -0.00027 & \multirow{2}{*}{0.71} \\
\hline & & & $\mathrm{S} 2$ & 0.19 & 0.014 & 0.00084 & \\
\hline \multirow{8}{*}{$\begin{array}{l}\text { Ordinary } \\
\text { subjects }\end{array}$} & \multirow{2}{*}{ Subject5 } & \multirow{2}{*}{62.7} & S1 & -0.85 & 0.045 & -0.00051 & \multirow{2}{*}{0.95} \\
\hline & & & $\mathrm{S} 2$ & 0.45 & 0.0069 & 0.049 & \\
\hline & \multirow{2}{*}{ Subject6 } & \multirow{2}{*}{81.1} & S1 & -0.65 & 0.38 & -0.24 & \multirow{2}{*}{0.02} \\
\hline & & & $\mathrm{S} 2$ & -0.45 & 0.0058 & 0.00076 & \\
\hline & \multirow{2}{*}{ Subject7 } & \multirow{2}{*}{55.4} & S1 & -0.83 & 0.049 & 0.00077 & \multirow{2}{*}{0.97} \\
\hline & & & $\mathrm{S} 2$ & 0.7 & 0.077 & 5.97 & \\
\hline & \multirow{2}{*}{ Subject8 } & \multirow{2}{*}{76.8} & S1 & -1.61 & 0.14 & -0.0022 & \multirow{2}{*}{0.093} \\
\hline & & & $\mathrm{S} 2$ & 0.6 & 0.066 & 0.39 & \\
\hline
\end{tabular}

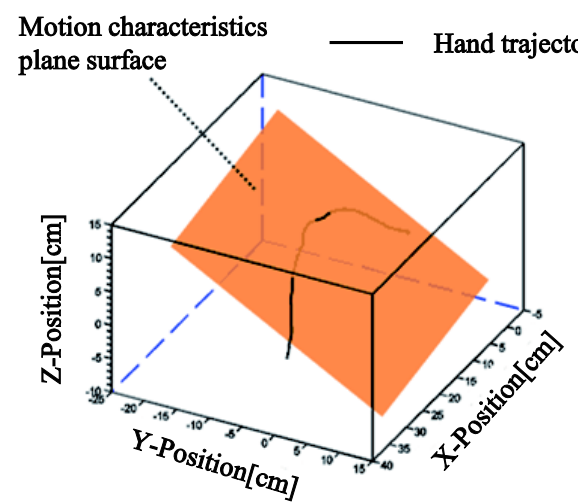

(a) Motion characteristics plane surface.

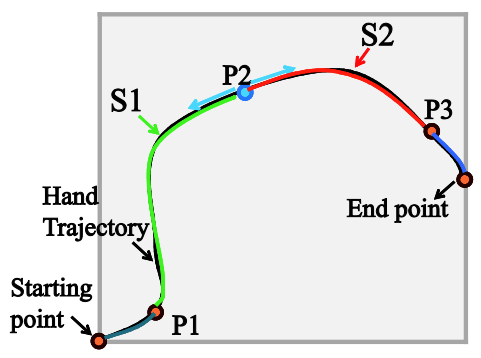

(b) Spline fitting.

Figure 4: $\quad$ Principal component analysis and spline fitting.

Fig. 5 shows the results of spline fitting for Subject1, Subject2, Subject5, and Subject7. The motions of Subject1 and Subject2 have an inflection point, and the shape of the trajectory changes from concave down to concave up from this point; these trajectories are considered S-shaped. Therefore, we consider Hogarth's definition to be accurate. In contrast, the parameters of general motions do not show the same characteristics. 


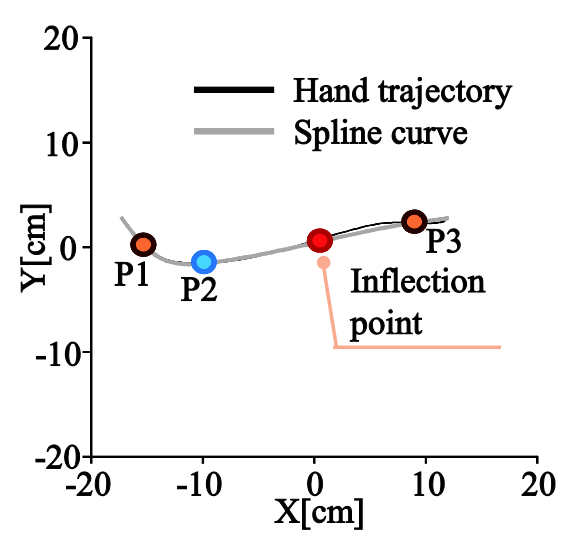

(a) Trajectory of Subject1.

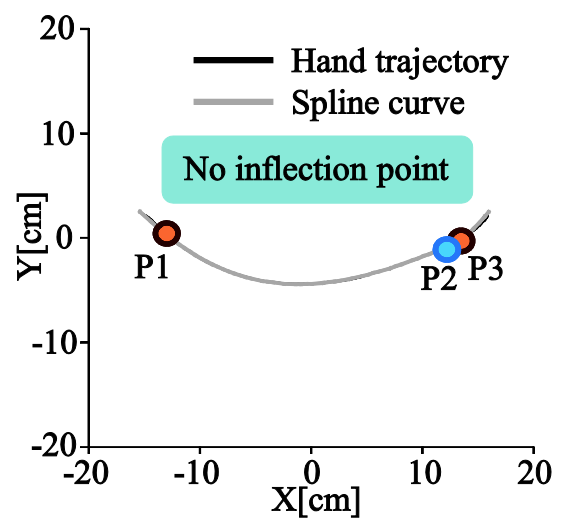

(c) Trajectory of Subject5.

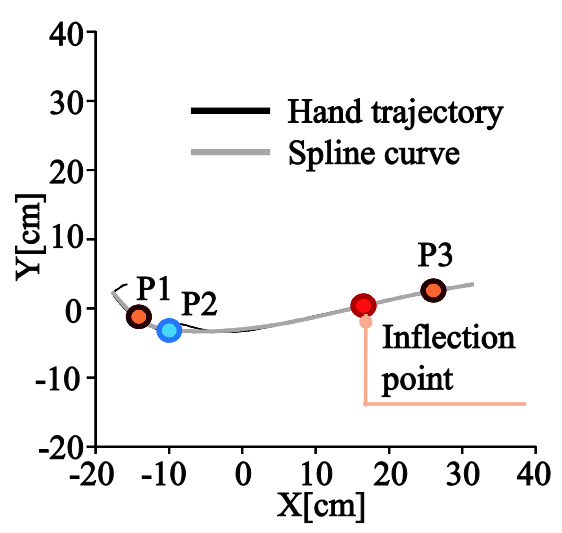

(b) Trajectory of Subject2.

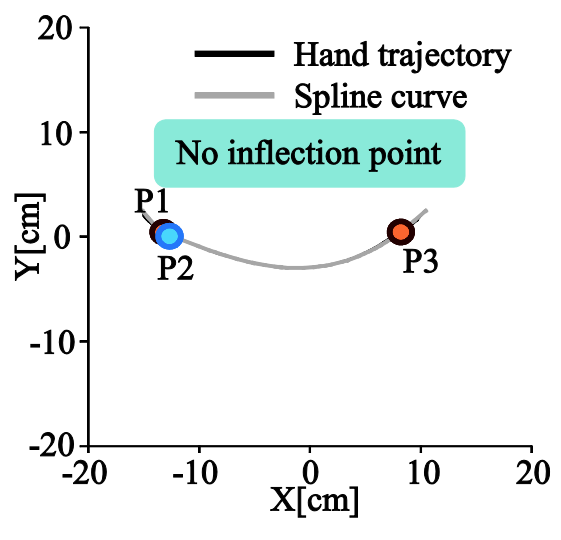

(d) Trajectory of Subject7.

Figure 5: $\quad$ Results of spline fitting.

\section{Simulation of graceful motions}

As mentioned previously, the characteristics of graceful motions were extracted as parameters of the spline curves and $\theta$ that is the angle between the motion characteristics plane surface and the $\mathrm{x}-\mathrm{z}$ plane. As a result, common characteristics were confirmed in graceful motions. However, the parameters of graceful motions were not obtained correctly. Therefore, we changed the extracted parameters step by step and verified which parameters provide graceful impressions through simulations. 


\subsection{Conditions of simulations}

In this study, a simulation was conducted by changing the parameters of Subject1, whose impressions were evaluated to be the most favorable ones. The $\theta$ value was changed step by step and 4 motions were created using 3DCG. Table 3 shows the changed parameters. In this case, the $b, c$, and $d$ values were multiplied by five to highlight the differences. Fig. 6 shows Motion1 and Fig. 7 shows the other motions. The white arrow and the white line indicate the direction of motion and the trajectory of motion respectively.

Table 3: $\quad$ Changed parameters.

\begin{tabular}{|c|c|c|c|c|c|}
\hline & $\theta\left[^{\circ}\right]$ & $\mathrm{S}$ & $b$ & $c$ & $d$ \\
\hline \multirow{2}{*}{ Motion1 } & \multirow{2}{*}{45} & $\mathrm{~S} 1$ & -5.495 & 1.02 & -0.057 \\
\cline { 3 - 6 } & & $\mathrm{S} 2$ & 0.56 & 0.565 & -0.00205 \\
\hline \multirow{2}{*}{ Motion2 } & \multirow{2}{*}{90} & $\mathrm{~S} 1$ & -5.495 & 1.02 & -0.057 \\
\cline { 3 - 6 } & & $\mathrm{S} 2$ & 0.56 & 0.565 & -0.00205 \\
\hline \multirow{2}{*}{ Motion3 } & \multirow{2}{*}{135} & $\mathrm{~S} 1$ & -5.495 & 1.02 & -0.057 \\
\cline { 3 - 6 } & & $\mathrm{S} 2$ & 0.56 & 0.565 & -0.00205 \\
\hline \multirow{2}{*}{ Motion4 } & \multirow{2}{*}{270} & $\mathrm{~S} 1$ & -5.495 & 1.02 & -0.057 \\
\cline { 3 - 6 } & & $\mathrm{S} 2$ & 0.56 & 0.565 & -0.00205 \\
\hline
\end{tabular}

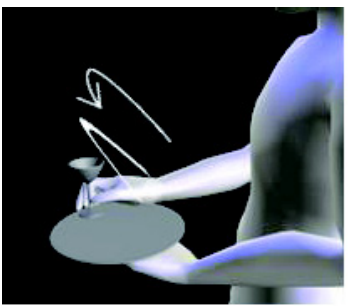

(1)

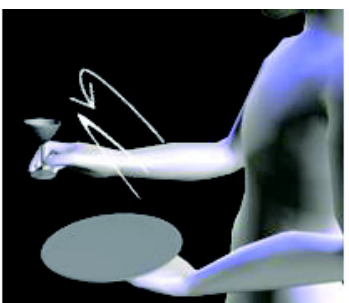

(2)

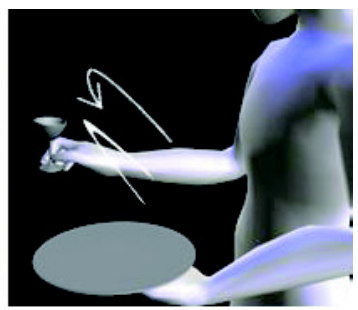

(3)

Time

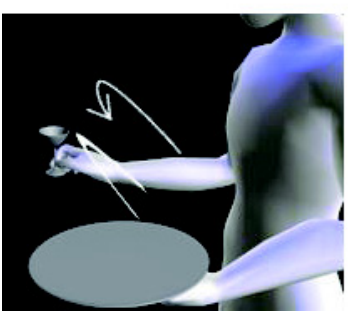

(4)

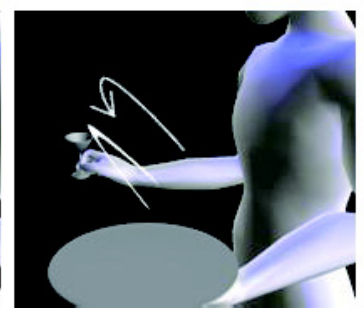

(5)

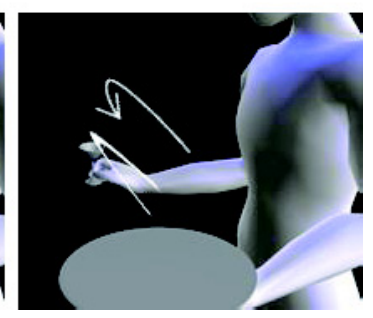

(6)

Figure 6: Motion1. 

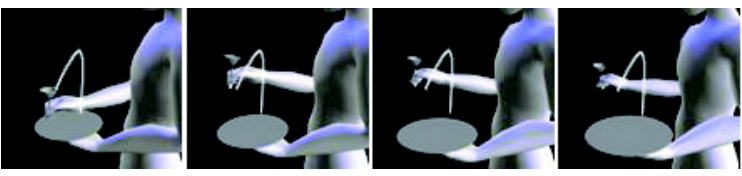

(a) Motion2
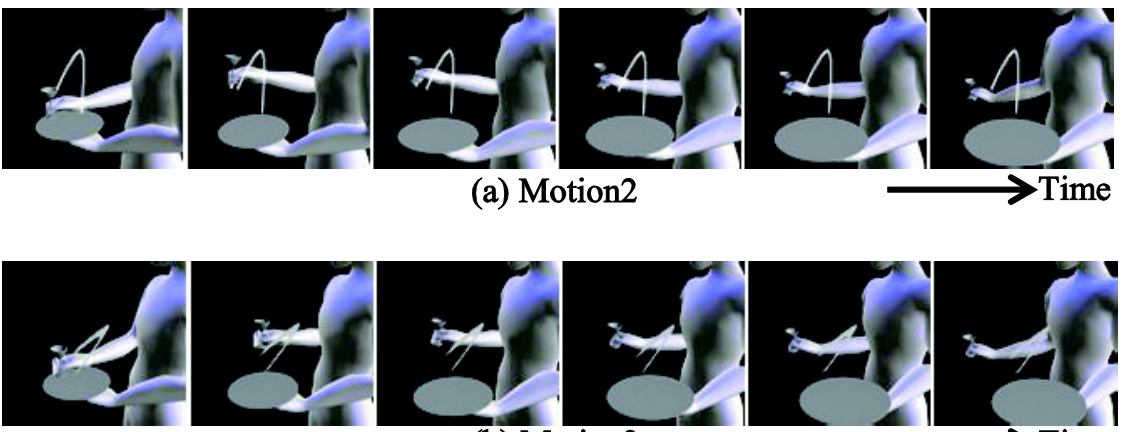

(b) Motion3
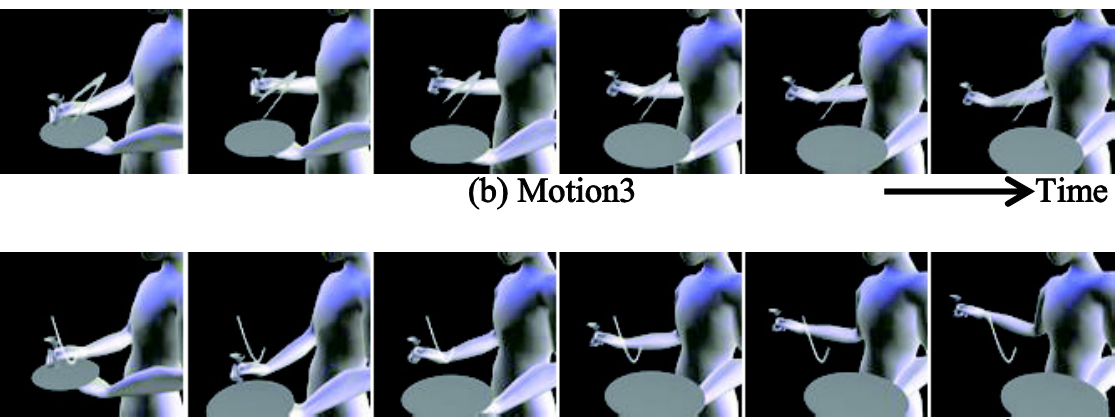

(c) Motion4

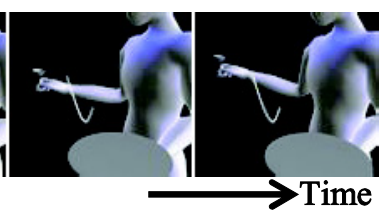

Figure 7: $\quad$ Created motions.

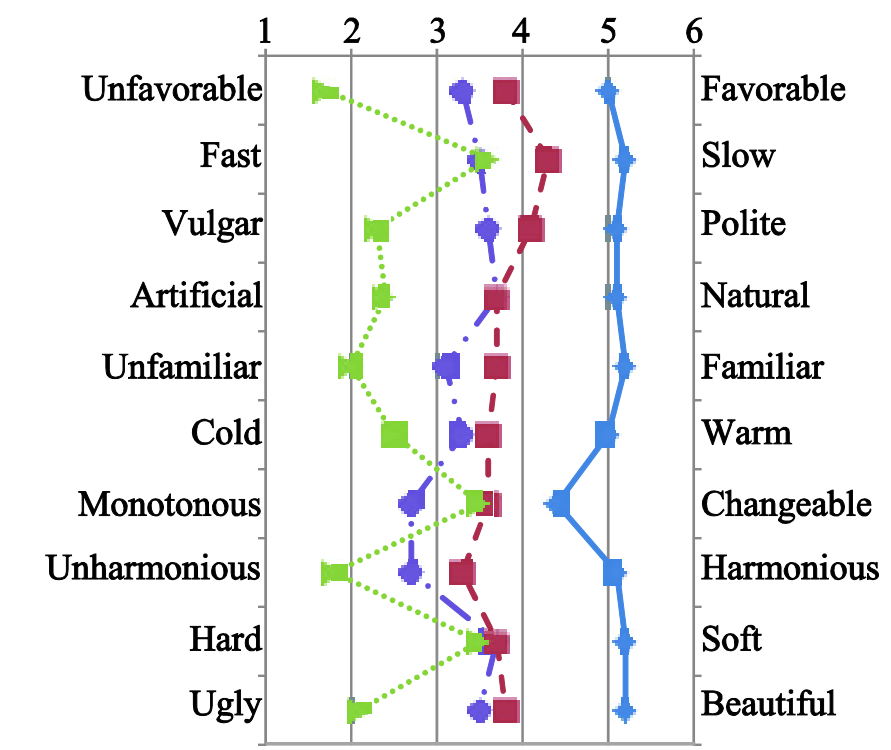

$\sim$ Motion1 $\longrightarrow$ Motion2 $-\square$ Motion3 … Motion4

Figure 8: $\quad$ SD profile. 


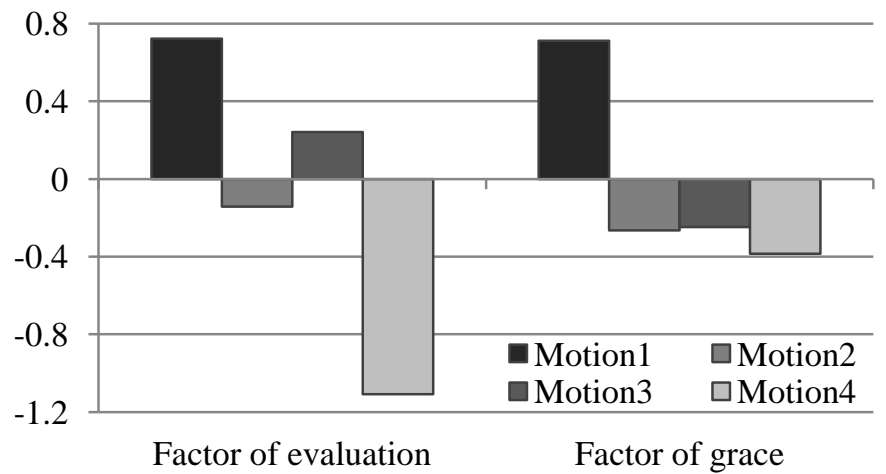

Figure 9: $\quad$ Factor scores.

\subsection{Evaluation of impressions}

Four motions were created and shown to ten observers, and they were evaluated against 10 pairs of adjectives. Fig. 8 shows the SD profile. As shown in this profile, the evaluations of Motion2 and Motion4 are poor. This result suggested that motions with $\theta$ values of $90^{\circ}$ and $270^{\circ}$ produced unfavorable impressions. On the other hand, the evaluation of Motion3 was higher than that of Motion2 and Motion4. Moreover, the evaluation of Motion1 was the best. This result suggested that motions performed in crosswise directions are better than those performed in vertical directions; furthermore, motions with the $\theta$ value of $45^{\circ}$ produced favorable impressions.

\subsection{Factor analysis}

After the evaluation of the impressions, factor analysis was conducted, from which two factors were extracted. One factor was called the factor of evaluation because its factor loadings show high values for the adjectives Pleasant, Warm, and Familiar. The other factor was called factor of grace because its factor loadings show high values for the adjectives Tender, Beautiful, and Natural. Fig. 9 shows each factor score. Motion 1 shows the highest scores, and motion with a $\theta$ value of $45^{\circ}$ produce favorable and graceful impressions.

\section{Conclusion}

In this study, we analyzed the handover motion of a glass to quantify the characteristics of graceful motions from human motions. The result showed that graceful motions are characterized by S-shaped trajectories. One of the parameters is the $\theta$ value, and values that produced favorable were determined through simulations.

We intend to obtain a range of parameters that produce graceful motions by carefully changing $\theta$, with a focus on a $\theta$ value of $45^{\circ}$. We also intend to conduct a simulation by changing the values of $b, c$, and $d$. Finally, we intend to analyze 
motions other than the handover motion and extract the characteristics of graceful motions.

\section{Acknowledgement}

This work was supported by JSPS KAKENHI Grant Number 23500249.

\section{References}

[1] Abu bakar, S., Ikeura, R., Handa, Y., Yano, T., Mizutani, K. and Sawai, H., Communucation during the Cooperative Motion in the Task of Carrying an Object between Two Humans, Journal of Biomechanical Science and Engineering, Vol. 5, No. 2, pp. 104-118, 2010

[2] Yokoi, K., Arisumi, H. and Komoriya, K., Gentle Motion of Robot Manipulator - How does human feel hand-over motion of a robot? - Journal of Mechanical Engineering Laboratory, Vol. 52, No. 4, pp. 149-155, 1998

[3] Meinel, K., Asthetik der Bewegung, Taishukan Publishing Co., Ltd., 1998. (in Japanese)

[4] Schiller, F., "Ueber Anmut und Wuerde” 1794. (Nagakura, S., Aesthetic Consideration of Humans, Michitani Publishing, 2003. (in Japanese))

[5] Hogarth, W., The Analysis of Beauty, Chuokouronbijyutsu Publishing, 2007. (in Japanese)

[6] Buytendijk, F.J.J., Allgemeine Theorie der Menschlichen Haltung und Bewegung, Springer 1972

[7] Tsuduki, T., Tanaka, T. and Ueda, E., Extraction of graceful motion features from handing task, Proceedings of ROBOMEC 2012, 2A1-Q05, 2012 